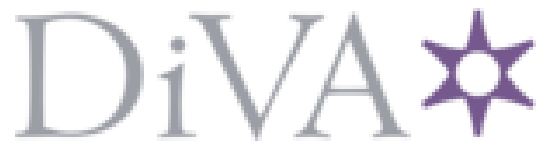

http://www.diva-portal.org

Preprint

This is the submitted version of a paper presented at HRI'2O: ACM/IEEE International Conference on Human-Robot Interaction, Cambridge-United Kingdom, March 23-26, 2020.

Citation for the original published paper:

Calvo Barajas, N., Elgarf, M., Perugia, G., Peters, C., Castellano, G. (2020)

Can a Social Robot Be Persuasive Without Losing Children's Trust?

In: HRI '2O: Companion of the 2020 ACM/IEEE International Conference on HumanRobot Interaction (pp. 157-159). New York, NY, United States: ACM Digital Library https://doi.org/10.1145/3371382.3378272

N.B. When citing this work, cite the original published paper.

Permanent link to this version:

http://urn.kb.se/resolve?urn=urn:nbn:se:uu:diva-408953 


\section{Can a Social Robot Be Persuasive Without Losing Children's Trust?}

\author{
Natalia Calvo \\ natalia.calvo@it.uu.se \\ Uppsala University \\ Uppsala, Sweden \\ Maha Elgarf \\ mahaeg@kth.se \\ KTH Royal Institute \\ of Technology \\ Stockholm, Sweden
}

\author{
Giulia Perugia \\ giulia.perugia@it.uu.se \\ Uppsala University \\ Uppsala, Sweden
}

\author{
Christopher Peters \\ chpeters@kth.se \\ KTH Royal Institute \\ of Technology \\ Stockholm, Sweden
}

\author{
Ginevra \\ Castellano \\ ginevra.castellano@it.uu.se \\ Uppsala University \\ Uppsala, Sweden
}

\begin{abstract}
Social robots can be used to motivate children to engage in learning activities in education. In such contexts, they might need to persuade children to achieve specific learning goals. We conducted an exploratory study with 42 children in a museum setting. Children were asked to play an interactive storytelling game on a touchscreen. A Furhat robot guided them through the steps of creating the character of a story in two conditions. In one condition, the robot tried to influence children's choices using high-controlling language. In the other, the robot left children free to choose and used a low-controlling language. Participants in the persuasive condition generally followed the indications of the robot. Interestingly, the use of high-controlling language did not affect children's perceived trust towards the robot. We discuss the important implications that these results may have when designing children-robot interactions.
\end{abstract}

\section{KEYWORDS}

Trust; Human-Robot Interaction; Persuasion; Reactance

\section{ACM Reference Format:}

Natalia Calvo, Maha Elgarf, Giulia Perugia, Christopher Peters, and Ginevra Castellano. 2020. Can a Social Robot Be Persuasive Without Losing Children's Trust?. In Companion of the 2020 ACM/IEEE International Conference on Human-Robot Interaction (HRI '20 Companion), March 23-26, 2020, Cambridge, United Kingdom. ACM, New York, NY, USA, 3 pages. https: //doi.org/10.1145/3371382.3378272

\section{INTRODUCTION}

In education, social robots have been used as peers or companions due to their effectiveness in eliciting learning outcomes [2]. In this context, the robot might have the role of giving advices or suggestions to influence childrens' behaviors and attitudes to achieve a specific goal. For instance, a persuasive social robot can motivate children to eat more vegetables and fruits [1]

Persuasion is the conscious intention to persuade or convince another person to change or maintain a particular action [9]. When humans face persuasive attempts, they can respond by showing compliance (e.g., act according to a suggestion) or reactance (e.g., resistance to follow a suggestion). Psychological studies have shown

Permission to make digital or hard copies of part or all of this work for personal or classroom use is granted without fee provided that copies are not made or distributed for profit or commercial advantage and that copies bear this notice and the full citation on the first page. Copyrights for third-party components of this work must be honored. For all other uses, contact the owner/author(s).

HRI '20 Companion, March 23-26, 2020, Cambridge, United Kingdom

(c) 2020 Copyright held by the owner/author(s)

ACM ISBN 978-1-4503-7057-8/20/03.

https://doi.org/10.1145/3371382.3378272 that children are prone to psychological reactance in response to persuasive communication as this constitutes a threat to their perceived freedom [3]. This reactance is higher especially towards authority figures such as parents and mentors [9]. Explicit messages are clear and show the real intention and are associated with high-controlling language, while implicit messages convey multiple meanings and interpretations and are associated with lowcontrolling language [8]. Besides, similarity and trustworthiness can reduce resistance against persuasive communication. For instance, [12] found that trust is mediated by similarity: when people perceived the communication source as more similar to theirs, they were more likely to trust it and less reactive to it.

In Human-Robot Interaction (HRI), factors like voice, gestures, and gaze may influence the persuasiveness of a robot $[4,6,11]$. In [5], a persuasive robot with a high-level of interactive social cues elicited lower psychological reactance compared to a robot with a low-level of social cues.

In this exploratory study, we aim to investigate whether a social robot that uses high-controlling language can i) persuade children to follow its lead when creating a storytelling character, and ii) can influence the perception of trustworthiness. To do so, we designed an interactive storytelling game in which children created a character with the help of a social robot. We used Furhat, a back-projected human-like robot head that conveys facial expressions in a smooth way [10].

\section{METHODS}

\subsection{Design and Measures}

We designed a between-subjects study with two experimental conditions (Persuasive vs. Neutral) to the independent variable Guidance. In the Persuasive condition, the robot tried to influence the user's choices using high-controlling language. To guide the child's behavior, it used persuasive statements, statements of praise (e.g., "I like your choice!") to approve the child's choice, and statements of disapproval (e.g., "This is not what I expected!") when the child choose a different option than the one suggested by the robot. In the Neutral condition, the robot followed up on the user's choices using low-controlling language. For both conditions, we implemented the same level of social cues for the robot (facial expressions of emotion and head movements). We defined four dependent variables: Compliance, that measures the number of times the child decided to follow the robot's guidance (this value was calculated only for the persuasive condition), Trustworthiness, that measures the child's trust towards the robot's advice and suggestions, Likability of the robot, and Enjoyment of the activity. 


\subsection{Participants}

We conducted the study at a science event for schools organized by the local Technical Museum. Children who visited the museum were asked to participate in the experiment. We did not collect personal data from the children's interaction with the robots but only asked them to fill out the questionnaires. Fifty-two children took part in the study, but only forty-two of them (17 female and 25 male), aged between 12 and 14 years $(M=12.09, S D=.50)$ completed the questionnaires.

\subsection{Apparatus and Stimuli}

The interaction consisted of an interactive-collaborative game between the child and the Furhat robot that aimed at creating the character of a story. The robot was remotely operated with a Wizard of $\mathrm{Oz}$ technique. The game was created using Unity and consisted of a number of steps through which the child could create the character. At each step of the game, the child could choose among a number of options available to customize the character. The options included a context (classroom or park), a main character to the story (adult, child, animal), features of the character (hair color, skin color, clothes, emotional expression for the human characters and an activity for the animals).

In the persuasive condition, the robot attempted to influence the children's choices three times: 1) in the choice of character, 2) in the choice of the hair color (human character) or in the choice of the animal type (animal character), and 3) in the choice of clothes (human character) or in the choice of the activity (animal character). The robot used explicit messages (e.g., "My flatmate has a cat, let's choose a cat") to influence the child's choice. In the neutral condition, the robot statements were neutral to guide the child either by explaining the next action or by asking about the child's preferences. Example statements included: "Now is the time to choose an outfit for the character!" and "Which color would you prefer for the character's hair?".

\subsection{Experimental Setup and Procedure}

The experimental setup consisted of the robot placed on a table in front of the child. The game was displayed on a touch screen situated between both of them.

The study was conducted in a small station built in an isolated corner of a room at the museum. The children arrived to the station and were recruited one by one to take part in the experiment. The assignment of children to conditions was random and not all the children that took part in the event participated in the study. The experimenter started by greeting the child and briefly explaining the interaction. The child then engaged in the game with the robot for a duration of 8-10 minutes. At the end of the interaction, the robot asked the child to fill a questionnaire on a tablet. The questionnaire included demographic data, measures of trust (trust in the robot's advice and trust in the robot's goodness), likability of the robot, and enjoyment of the activity [7]. After filling the questionnaire, the experimenter debriefed the participants and thanked them for participation. As participation in the experiment was voluntary, children could drop the activity at any time. Because of this, the conditions had a slightly different sample of participants $($ Persuasive $=23$, Neutral $=19)$.

\section{RESULTS}

In the Persuasive condition, $8.7 \%$ of participants $(N=2)$ never followed the suggestions of the robot, $21.7 \%$ of them $(N=5)$ followed the robot's lead once, $34.8 \%(N=8)$ followed it two times, and $21.7 \%$ of them $(N=5)$ all of the three times (missing data from 3 participants $=13 \%$ ). In general, as $78.2 \%$ of participants were persuaded by the robot at least once in the Persuasive condition, we can state that the manipulation of the persuasive condition was successful and proceed to further analyses.

We performed an independent t-test analysis with Guidance (Persuasive vs Neutral) as between-subjects variable on the variables trust robot's advice, trust robot's goodness, robot's likability, and enjoyment of the activity. Results revealed that the type of guidance did not affect trustworthiness in terms of robot's advice, $t(39)=-.389, \rho=.700$, nor in terms of robot's goodness, $t(39)=$ $-.482, \rho=.633$. Also, we found no significant effect on likability, $t(39)=-.840, \rho=.406$, nor enjoyment, $t(39)=-1.566, \rho=.125$. This suggests that the use of high-controlling language did not affect the children's perceived trustworthiness and likability toward the robot, nor was it detrimental to the enjoyment of the activity.

Further analysis of the descriptive statistics showed that in the Persuasive condition, $39.1 \%$ of the children perceived the robot as a stranger, $26.1 \%$ as a friend, $13 \%$ as a classmate, $4.3 \%$ as other, and $17.4 \%$ did not answer. Whereas in the Neutral condition, $42.1 \%$ of the children perceived the robot as a friend, $31.6 \%$ as a stranger, $5.3 \%$ as a classmate, $5.3 \%$ as a teacher, $5.3 \%$ as a relative, and $5.3 \%$ did not answer.

\section{DISCUSSION}

Experimental results presented in this paper suggest that a persuasive social robot that uses explicit language and interactive praise and disapproval may influence children's behaviors and attitudes. In agreement with previous studies, we found that children follow the robot's suggestions to achieve a goal [1]. Also, we found that high-controlling language does not affect the children's perceived trust in the robot. This result may be related to the role the robot has for the child. Literature suggests that children tend to have negative responses when they are subjected to persuasive communication from their parents or mentors [9]. Our results revealed that the robot was mostly perceived as a stranger, and, often, as a peer (e.g., a friend, a classmate). We believe that this role might have enhanced the robot's perceived trustworthiness and likability, and might have mitigated the reactance to the high-controlling language in the persuasive condition. It is interesting to note, however, that children perceived the robot as as stranger more often in the Persuasive condition with respect to the Neutral condition. These preliminary results pinpoint the importance of the robot's role and of the explicitness of its message to motivate children to complete a learning task, but also, the need to develop persuasive robots able to match the objective of the message, and the child's salient goal orientation in educational settings.

\section{ACKNOWLEDGMENTS}

This work was supported by the European Commission Horizon 2020 Research and Innovation Program under Grant Agreement No. 765955. 


\section{REFERENCES}

[1] Ilaria Baroni, Marco Nalin, Mattia Coti Zelati, Elettra Oleari, and Alberto Sanna 2014. Designing motivational robot: how robots might motivate children to eat fruits and vegetables. In The 23rd IEEE International Symposium on Robot and Human Interactive Communication. IEEE, 796-801.

[2] Tony Belpaeme, James Kennedy, Aditi Ramachandran, Brian Scassellati, and Fumihide Tanaka. 2018. Social robots for education: A review. Science robotics 3, 21 (2018), eaat5954.

[3] Jack W Brehm. 1966. A theory of psychological reactance. (1966).

[4] Arturo Cruz-Maya and Adriana Tapus. 2018. Negotiating with a robot: analysis of regulatory focus behavior. In 2018 IEEE International Conference on Robotics and Automation (ICRA). IEEE, 1-9.

[5] Aimi Shazwani Ghazali, Jaap Ham, Emilia Barakova, and Panos Markopoulos. 2019. Assessing the effect of persuasive robots interactive social cues on users' psychological reactance, liking, trusting beliefs and compliance. Advanced Robotics 33, 7-8 (2019), 325-337.

[6] Jaap Ham, Raymond H Cuijpers, and John-John Cabibihan. 2015. Combining robotic persuasive strategies: the persuasive power of a storytelling robot that uses gazing and gestures. International fournal of Social Robotics 7, 4 (2015)
479-487

[7] Marcel Heerink, Ben Kröse, Vanessa Evers, and Bob Wielinga. 2010. Assessing acceptance of assistive social agent technology by older adults: the almere model. International journal of social robotics 2, 4 (2010), 361-375.

[8] Elissa Lee and Laura Leets. 2002. Persuasive storytelling by hate groups online: Examining its effects on adolescents. American behavioral scientist 45, 6 (2002), 927-957.

[9] Claude H Miller. 2015. Persuasion and psychological reactance: The effects of explicit, high-controlling language. In The exercise of power in communication. Springer, 269-286.

[10] Samer AL Moubayed, Gabriel Skantze, and Jonas Beskow. 2013. The furhat back-projected humanoid head-lip reading, gaze and multi-party interaction. International fournal of Humanoid Robotics 10, 01 (2013), 1350005.

[11] Mikey Siegel, Cynthia Breazeal, and Michael I Norton. 2009. Persuasive robotics: The influence of robot gender on human behavior. In 2009 IEEE/RSf International Conference on Intelligent Robots and Systems. IEEE, 2563-2568.

[12] Hwanseok Song, Katherine A McComas, and Krysten L Schuler. 2018. Source Effects on Psychological Reactance to Regulatory Policies: The Role of Trust and Similarity. Science Communication 40, 5 (2018), 591-620. 